\title{
Anna BOCHNAKOWA
}

Université Jagellonne de Cracovie

http://dx.doi.org/10.18778/8088-785-5.19

\section{Les anglicismes avec food en polonais actuel}

\begin{abstract}
Résumé
L'article est consacré aux anglicismes composés avec food en polonais contemporain. Les exemples comfort food, fast food, food book, food cost, food court, food justice, food pairing, food porn, food truck, food writer, foodie, foodis, foodsharing, fudsejwer, good food, junk food, raw food, slow food, soul food, street food, superfood proviennent de la presse quotidienne et de l'Observatoire linguistique de l'Université de Varsovie (Obserwatorium Językowe UW). Pour pouvoir dater l'entrée des néologismes cités en polonais, la vérification dans le Corpus national de la langue polonaise (NKJP) a été effectuée.

Les mots répertoriés sont présentés dans leurs contextes (traduits en français) et accompagnés d'un commentaire concernant l'assimilation morphologique et sémantique en polonais. Les équivalents polonais, souvent des néologismes récents eux aussi, sont rapportés à côté de certains anglicismes.
\end{abstract}

Mots-clés : polonais, anglicismes, composés avec food

\section{Summary}

The paper discusses the compound words containing food borrowed from English. Examples (comfort food, fast food, food book, food cost, food court, food justice, food pairing, food porn, food truck, food writer, foodie, foodis, foodsharing, fudsejwer, good food, junk food, raw food, slow food, soul food, street food, superfood) come from newspapers and the Language Observatory of the University of Warsaw. In order to specify the date of cited neologisms, the verification in the National Corpus of Polish was carried out. 
The chosen words are presented in their contexts (translated into French) and annotated with morphological and semantic assimilation in Polish. Alongside some of the anglicisms the Polish counterparts, including the recent ones, appear.

Keywords: Polish language - anglicisms - compounds with food.

\section{Introduction}

Ayant pris connaissance de la contribution de Christine Jacquet-Pfau et de Zuzana Hildenbrand dans Emprunts néologiques ${ }^{1}$ consacrée aux emprunts en français et en tchèque dans le domaine des pratiques alimentaires contemporaines, nous nous sommes proposé de passer en revue les néologismes composés contenant le mot food, que nous avons repérés durant les six derniers mois (avril - septembre 2016) dans Gazeta Wyborcza et ponctuellement dans Dziennik Polski et l'hebdomadaire Polityka de la même période. Ont été inclus dans le corpus quelques exemples répertoriés dans l'Observatoire linguistique, créé il y a deux ans par l'Université de Varsovie.

L'objectif est la vérification de leur adaptation en polonais et la recherche de leurs équivalents polonais, s'ils existent. Voici la liste alphabétique des exemples : comfortfood, fast food, food book, food cost, food court, food justice, food pairing, food porn, food truck, food writer, foodie, foodis, foodsharing, fudsejwer, good food, junkfood, raw food, slow food, soul food, street food, superfood. Certains de ces mots ont servi à la formation des dérivés en polonais, d'autres ont déjà des équivalents autochtones.

La première démarche a été de chercher à établir la date de l'emprunt de ces mots. La tâche n'était pas simple et les résultats ne paraissent pas satisfaisants, faute de corpus systématiquement alimentés. Les données de NKJP ('Corpus national de la langue polonaise') s'arrêtent vers 2010. N’y figurent que quelques attestations.

En 2014, un groupe de linguistes de l'Université de Varsovie a fondé l'« Obserwatorium Językowe » ('Observatoire linguistique') qui se propose d'enregis-

1 Christine Jacquet-Pfau et de Zuzana Hildenbrand, 2016, «Les pratiques alimentaires, un domaine très ouvert aux emprunts ", in Emprunts néologiques et équivalents autochtones en français, en polonais et en tchèque, Zuzana Hildenbrand, Alicja Kacprzak, Jean-François Sablayrolles (éds.), Limoges, Lambert Lucas, p. 175-225. 
trer les néologismes apparus en polonais à partir de 2000 et entrés dans l'usage standard, mais qui ne figurent pas dans les dictionnaires, même ceux qui enregistrent et expliquent des mots d'origine étrangère. La liste des néologismes est constituée avec l'aide des internautes, les rédacteurs complètent les articles avec des informations métalinguistiques ${ }^{2}$. On y trouve la transcription phonétiquelisible pour les locuteurs polonais, la définition du sens, le mot dans le contexte et quelques exemples d'emploi. Les articles sont signés par l'auteur et le rédacteur et comportent la date de l'enregistrement dans l'Observatoire (pratiquement : 2015 et 2016), ce qui ne donne aucune indication sur le moment et les circonstances de l'entrée des lexèmes étrangers en polonais.

Dans la présentation des exemples réunis, est adopté l'ordre suivant: d'abord ceux qui figurent dans le NKJP, donc notés avant 2010 et considérés comme les plus anciens (1). Ils sont présentés dans l'ordre établi d'après le nombre d'attestations dans ce corpus; puis les exemples de l'Observatoire linguistique, s'ils n'apparaissent pas dans le NKJP (2), et enfin les exemples relevés pour servir d'illustration à cette communication et qui ne sont notés ni dans le NKJP, ni dans l'Observatoire de Varsovie ${ }^{3}$ (3).

\section{Les emprunts avec food notés dans le NKJP : fast food, slow food, junk food, food court, superfood 4}

Fast food est attesté dans 122 contextes, ce qui rend ce mot le mieux documenté parmi les exemples. Les premiers textes proviennent du début des années 90 du siècle passé $(1992,1993)$ et apparaissent d’une façon ininterrompue dans la première décennie du XXIe siècle. La définition la plus simple est donnée dans un des contextes du corpus : il s'agit d'une conception de la restauration servant rapidement de la nourriture peu coûteuse, développée comme solution au

2 Le registre des mots commentés est accessible sur le site de l'Observatoire : http:// nowewyrazy.uw.edu.pl/

3 L'exemplier fourni à l'assistance lors du Colloque "Emprunts néologiques et équivalents autochtones. Mesure de leur circulations respectives " (octobre 2016 à Łódź) contenait ces derniers, dans l'ordre alphabétique, placés dans leur contexte. Je les ai cités indépendamment de leur présence dans le NKJP ou sur les listes de l'Observatoire.

4 Nous les avons tous repérés dans la presse écrite en 2016. 
principal point faible de la restauration traditionnelle : la durée trop longue de la préparation et du service. La liste de l'Observatoire linguistique ne contient pas ce mot, ses rédacteurs ne doivent plus le considérer comme un néologisme. Le terme paraît être bien acclimaté en polonais, bien qu'il garde le plus souvent la graphie anglaise, à ceci près que les deux éléments peuvent paraître liés. La prononciation est quasi originale. On rencontre des attestations de la flexion - formes des cas obliques et du pluriel. Le sens du lexème est double : 'préparations culinaires assez simples et bon marché, pouvant aussi être emportées' et aussi 'restaurant, bar, etc. où ce type de cuisine est pratiqué et servi, souvent appartenant à une chaîne de restauration'. À ce dernier sens correspond le polonais bar szybkiej obstugi ('bar à service rapide'), courant dans l'usage. Depuis une dizaine d'années environ, on peut trouver des contextes où fast food porte une nuance péjorative, qui vient certainement des courants promouvant la nourriture saine, voire biologique, et une certaine culture de la consommation. En Pologne, après l'ouverture du pays aux influences occidentales, les nouveautés gastronomiques étrangères ont été accueillies 5 avec enthousiasme et positivement, et, depuis un certain temps, d'un côté elles commencent à avoir mauvaise presse et de l'autre, avec l'emploi des termes étrangers, anglais pour la plupart, elles restent un emblème de l'intégration de la Pologne dans le monde occidental.

S'est présenté récemment un emploi réhabilitant en quelque sorte le fast food. Dans le concours organisé pour les restaurateurs « Les goûts de Cracovie », on a distingué une catégorie 6 : «Szybka pycha - fast food z klasą » ('gourmandise rapide - fast food classe'), où le mot pycha (ici : 'plat savoureux') remplace l'anglais food et le commentaire valorise la notion, généralement prise aujourd'hui comme négative.

Le substantif masculin fast food a servi en polonais à la formation de l'adjectif fastfoodowy, décliné lui aussi, comme dans l'exemple suivant : « Nadgorliwe kucharki mylnie interpretowały założenia „tarczy antyfastfoodowej” " ('Les cuisinières trop zélées ont mal interprété les principes de «bouclier antifastfood $)^{7}$.

5 Voir à ce propos : Anna Bochnakowa, Zuzana Hildenbrand, 2016, « Des néologismes culinaires récents en polonais et en tchèque ", in Emprunts néologiques et équivalents autochtones en français, en polonais et en tchèque, Zuzana Hildenbrand, Alicja Kacprzak, Jean-François Sablayrolles (éds.), Limoges, Lambert Lucas, p. 227-266.

6 Dziennik Polski, le 27 août 2016 : Plebiscyt Smaki Krakowa (...).

7 Wysokie Obcasy, le 27 août 2016. 
Le néologisme polonais fastfoodztwo, figurant dans l'Observatoire linguistique, peut servir d'illustration, semble-t-il, d'une nuance négative de fast food qui est à l'origine de sa formation. Voici la définition donnée par l'Observatoire : fastfoodztwo " jedzenie typu fast food postrzegane jako szczególnie nieapetyczne i niezdrowe " ('nourriture de type fast food perçue comme particulièrement dégoûtante et malsaine'), et quelques exemples provenant des sites internet : « Gdy jestem gdzieś poza domem i głód mnie dopadnie, to wolę słodką bułkę z piekarni niż jakieś fastfoodztwo (wizaz.pl)» ('Quand je suis hors de chez moi et que j'ai une faim subite, je préfère une pâtisserie sucrée à un fastfoodztwo quelconque'), ou encore: «Zdajemy sobie sprawę jak nie ze szkodliwości, to przynajmniej z niezdrowości fastfoodztwa (chusty.info) » ('Nous nous rendons compte, sinon de la nuisibilité de fastfoodztwo, au moins de ses propriétés malsaines'). Ce néologisme fait penser à la contamination de l'expression anglaise et du mot polonais paskudztwo, substantif formé à partir de paskudny 'dégoûtant, moche, horrible'. Le principe de la formation repose sur l'identité de prononciation de « oo » anglais et de « $\mathrm{u}$ » polonais.

Le mouvement et le terme slow food apparaissent en Italie en 1986. Quand le fast food commence à prendre une nuance sémantique négative à cause de la qualité de la nourriture servie sous cette enseigne, le mouvement slow food est né (le nom se construit sur une antonymie) et il prend pour logo une image d'escargot, soulignant encore l'opposition à la rapidité suggérée dans fast food.

Slow food figure dans le NKJP dans 22 exemples, dont les plus anciens datent de 2003. L'Observatoire linguistique le répertorie aussi, avec la prononciation [slou fud] et la définition suivante : « ruch, którego celem jest zachowanie smaku potraw kuchni regionalnych świata oraz promowanie regionalnej, naturalnej żywności i metod jej wytwarzania » ('mouvement qui a pour l'objectif la préservation du goût de la cuisine régionale dans le monde et la promotion de la nourriture régionale, naturelle et des méthodes de sa production'). Le terme emprunté n'a pas d'équivalents polonais, il se plie pourtant à la flexion nominale : voir filozofia slow foodu 'philosophie du slow food' (génitif), o slow foodzie 'de slow food' (locatif), où la consonne finale du mot anglais subit une alternance comme dans le polonais powód/powodu/powodzie. On relève aussi des attestations de l'adjectif dérivé slowfoodowy. On trouve aussi une formule (titre d'un paragraphe d'un article dans Polityka du 13 mai 2016) paradoxale : szybki slow food ('slow food rapide').

Le terme junk food apparaît dans le NKJP dans 7 contextes. Le plus ancien (sur papier) date de l'an 2000, et il concerne l'emploi de l'expression en anglais, mais apporte un équivalent polonais surprenant : «W języku angielskim zagościło określenie junk food (fajansiarskie żarcie), oznaczające wysokokaloryczne, 
niezdrowe potrawy z barów szybkiej obsługi typu McDonald czy Burger King " ('Est apparu en anglais le terme junk food (fajansiarskie żarcie - 'malbouffe' ?) qui désigne des plats très caloriques et malsains provenant des fast food du type McDonald's ou Burger King'). Les trois attestations sur papier datent de 2008 et viennent d'un seul ouvrage ${ }^{8}$. La traduction de junk food pour fajansiarskie jedzenie semble être une création des auteurs de la publication ${ }^{9}$; dans l'usage courant on trouve śmieciowe jedzenie, calqué sur l'anglais. L'Observatoire linguistique ne note pas junkfood, alors que ce mot a une forte présence sur internet (plus de 250 millions d'apparitions, essentiellement dans Wikipedia version polonaise, mais dans des contextes anglais). Son équivalent polonais śmieciowe jedzenie a presque quinze mille attestations sur internet.

Le composé food court apparaît 3 fois dans le NKJP (2005-2007). Dans la plus ancienne attestation ${ }^{10}$, le mot est suivi d'une explication de sens et précédé par le mot czyli 'c'est-à-dire' qui signale le caractère néologique du mot anglais en polonais : «Na parterze natomiast będzie tzw. food court $\left(700 \mathrm{~m}^{2}\right)$ czyli wydzielone miejsce gdzie można zjeść coś szybko » ('Au rez-de-chaussée il y aura ce qui s'appelle un food court, c'est-à-dire un endroit isolé où l'on peut prendre rapidement quelque chose à manger'). Les citations datant de 2006 et de 2007 contiennent ce mot sans aucune marque typographique particulière. Food court figure aussi dans l'Observatoire linguistique, les rédacteurs de l'article donnent la prononciation du mot [futcort] et deux citations venant de sites internet.

Superfood (ou superfoods) désigne un produit de haute concentration en éléments nutritifs. Nous n'avons pas trouvé de formes déclinées de ce nom, mais une telle adaptation est possible, ainsi que la formation de l'adjectif. Dans les deux exemples ${ }^{11}$ l'emprunt garde le singulier là où le pluriel anglais ou polonais s'imposerait :

Jarmuż zaliczany jest do superfood, czyli produktów o wyjątkowych wartościach odżywczych " ('Le chou frisé est compté parmi les superfoods, c'est-à-dire les produits de valeurs nutritives exceptionnelles') ; "Matcha jest na liście superfood, czyli produktów o skoncentrowanej dawce składników odżywczych »

8 E. Rozumowski, Reiki: naturalna metoda uzdrawiania przez nakładanie rąk, éd. Kos, 2008.

9 Sto płyt, które wstrzasnęly światem, Grzegorz Brzozowicz, Filip Łobodziński, Iskry, Warszawa, 2000.

10 Dziennik Zachodni du 17 février 2005.

11 Gazeta Wyborcza - Tylko Zdrowie, le 3 février 2016; Gazeta Wyborcza - Tylko zdrowie, le 7 octobre 2016. 
('Le thé matcha se trouve sur la liste des superfoods, c'est-à-dire des produits avec une dose d'éléments nutritifs concentrée'). (Gazeta Wyborcza - Tylko Zdrowie, le 3 février 2016; Gazeta Wyborcza - Tylko zdrowie, le 7 octobre 2016.)

Le NKJP note un seul exemple d'emploi du mot superfood, en 2005, dans un bimensuel sur internet : «Polski przebój eksportowy robi furorę na świecie jako «superfood » i roślinna « viagra » ('Le hit polonais d'exportation fait fureur dans le monde en tant que « superfood » et " viagra » végétal')12. Le mot garde sa forme anglaise et il reste invariable. L'Observatoire linguistique a enregistré superfood et propose des équivalents : superjedzenie et superżywność attestés dans les ressources en ligne), calqués sur l'anglais.

Les autres mots de notre liste établie à partir d'une sélection de journaux de 2016 ne sont pas recensés dans le NKJP, qui, rappelons-le, n’est plus mis à jour depuis 2010.

\section{Le registre de l'Observatoire linguistique note les mots suivants ${ }^{13}$ : *comfort food, foodbook, foodpairing, *food porn, *food truck, *foodie, foodis ${ }^{14}$}

Comfort food (trois exemples dans le corpus papier) figure sur la liste de l'Observatoire avec la définition suivante : « posiłek przywołujący miłe wspomnienia, kojarzący się z domem i dzieciństwem, zwykle zawierający dużą liczbę kalorii i łatwy w przygotowaniu » ('plat évoquant des souvenirs agréables, en rapport avec la maison natale et l'enfance, généralement riche en calories et facile à préparer').

12 Dans notre corpus, nous avons encore attestation : " Myślenie o polish [sic] superfoods bywa wzmacniane świadomością ekonomiczną " ('L'idée de Polish superfoods est parfois renforcée par une conscience économique') dans Gazeta Wyborcza - Duży Format, le 25 mai 2016.

13 Nous marquons avec un astérisque les mots trouvés aussi dans la presse imprimée en 2016.

14 http://nowewyrazy.uw.edu.pl, je marque avec un astérisque les mots trouvés aussi dans la presse imprimée en 2016. 
Les rédacteurs notent le pluriel comfort foody et les formes avec le deuxième élément décliné : comfort foodu et comfort fooda (génitif), comfort foodzie (locatif). Les formes des épithètes dans les contextes suggèrent tantôt le genre neutre, tantôt le genre masculin du substantif emprunté. Dans les trois exemples du corpus papier le terme est mis entre guillemets. Les exemples de l'Observatoire provenant de sites internet notent comfort food sans guillemets. L'Observatoire note foodbook avec l'explication suivante: « wpis na blogu lub film wideo zawierający zdjęcia bądź nagrania wszystkich potraw, które autor zjadł danego dnia » ('note de blog ou film vidéo avec des photos des plats que l'auteur a mangés le jour même). Larticle cite aussi des formes du génitif du composé, écrites conjointement et trahissant une incertitude : foodbooka, foodbooku. Sont aussi notées les formes au pluriel foodbooki, foodbookach. Les citations proviennent de sources internet.

Foodpairing avec la prononciation indiquée [fudpering] est accompagné de la définition suivante : «w gastronomii technika łączenia różnych składników w celu tworzenia nieoczywistych połączeń smakowych » ('en gastronomie, une technique de combinaison d'ingrédients, visant à créer une composition de goûts originale et inattendue').

Le food porn est défini (en polonais, évidemment) par les rédacteurs de l'Observatoire comme 'des plats photographiés ou filmés d'une façon alléchante et sensuelle'. Le terme a intégré la flexion polonaise, le second élément peut prendre des formes de cas obliques : food porna (génitif), food pornem (instrumental), (o) food pornie (locatif) parfois écrit en un seul mot et utilisé aussi au pluriel foodporny. On rencontre enfin l'adjectiffoodpornowy, décliné lui aussi.

Le mot fréquent (dans notre inventaire d'exemples) food truck/foodtruck 'camionnette adaptée à la préparation et à la vente des plats chauds' a dans l'Observatoire ses équivalents polonais composés: jadłobus et jadłowóz, calqués sur le mot anglais, où food est traduit par jadło 'nourriture', forme archaïsante, sans doute quelque peu ironique, aujourd'hui considérée comme littéraire ${ }^{15}$ et de bus (aphérèse d'autobus) ou de wóz 'voiture' dans le second cas. Nous avons trouvé aussi le néologisme gastrobus donné comme équivalent de food truck. Le terme anglais se voit intégré dans la déclinaison (genre masculin) - foodtruckiem, foodtrucków, foodtruckach (les trois formes ici dans un emploi au pluriel). Parmi les exemples, on peut trouver également des adjectifs, déclinés eux aussi : foodtruckowych, foodtruckowa, etc. Il est à noter que foodtruck a servi à la for-

$15 \mathrm{Au}$ moins d'après Wielki słownik jezyka polskiego, http://www.wsjp.pl/index. php?id_hasla=20823\&ind=0\&w_szukaj=jad\%C5\%82o. 
mation des néologismes : foodtrackowiec (avec l'assimilation graphique due à la prononciation) 'propriétaire/vendeur de foodtruck' et foodtrucker, de même sens. Nous avons constaté avec intérêt la présence de deux formes sur la liste de l'Observatoire : foodie ${ }^{16}$ (nom anglais, pris comme masculin, invariable) 'ktoś, kto lubi jeść i ma dużą wiedzę na temat kulinariów' ('personne qui aime manger et qui a un vaste savoir dans le domaine culinaire') et foodis 'personne trouvant du plaisir et de la satisfaction dans la consommation de la nourriture saine et naturelle'). Dans les citations on a aussi foodisa (génitif) et foodisi au pluriel (nominatif). Le mot foodis semble être une adaptation du pluriel anglais foodies ${ }^{17}$, mais il est considéré en polonais comme un substantif masculin au singulier. Notons aussi la différence sémantique entre les deux emprunts : foodie reste près du sens anglais 'gourmet' alors que le sens de foodis 'amateur de la nourriture naturelle' est une innovation polonaise.

\section{Exemples tirés de la presse (non spécialisée) qui ne figurent ni dans le NKJP ni dans les registres de l'Observatoire linguistique de l'Université de Varsovie}

Fudsejwer (de foodsaver, que nous n'avons pas repéré) est un emprunt phonétique, trouvé une seule fois ${ }^{18}$ : « Ktoś dał lodówkę, zgłosiło się 20 fudsejwerów [en italique dans le texte] chętnych do dbania, żeby się nic nie zmarnowało » ('Quelqu'un a offert un frigidaire, 20 foodsavers se sont présentés prêts à veiller à ce que rien ne se perde'). Le sens du mot s'y trouve indirectement expliqué, et dans la suite du texte : « Ratownicy po sześćdziesiątce » voulant dire 'sauveurs de plus de 60 ans' fait allusion à l'anglais foodsaver et à l'âge des bénévoles. L'emprunt prend ici la forme du génitif pluriel exigée par la syntaxe de la phrase en polonais. Le genre masculin attribué à fudsejwer résulte de la finale conso-

16 Trouvé aussi dans Polityka du 13 mai 2016 : « [...] prędzej spróbuje jej [jagnięciny] włoski foodie [...] niźli krakus. [...] » ('il est plus probable qu’un foodie italien goûte de l'agneau [polonais] qu'un habitant de Cracovie').

17 Les rédacteurs de l'article notent l'exemple suivant : "Warszawscy foodies podążają za modą. Są zawsze jednymi z pierwszych klientów w nowych knajpach (hiro.pl) » ('Les foodies de Varsovie suivent la mode. Ils sont toujours parmi les premiers clients des restaurants récemment ouverts').

18 Polityka du 12 octobre 2016. 
nantique de l'anglais foodsaver. Foodsharing est aussi un emprunt récent en polonais : les attestations en ligne proviennent du début du mois de mai 2016, date à laquelle l'idée du partage de la nourriture est arrivée en Pologne, d'abord à Varsovie, puis dans d'autres villes. Nous l'avons trouvé seulement dans deux passages dans la presse imprimée ${ }^{19}$, mais accompagné à chaque fois de l'équivalent polonais jadłodzielnia, traduction de l'anglais, utilisé déjà dans les premières attestations en ligne.

Food cost apparaît une seule fois ${ }^{20}$ parmi les contextes que nous avons relevés, il est pourtant présent sur internet. Sur le site http://dla-gastronomii.pl/ kalkulator-food-cost, par exemple, on trouve la définition du terme : " Food cost to prosty, łatwy a zarazem niezwykle skuteczny sposób na obliczenie kosztu surowców zużytych do przygotowania potraw " ('Le food cost est un moyen simple, facile et efficace à la fois d'évaluer le coût des ingrédients utilisés pour la préparation de plats'), ou encore : « Food cost to bowiem bezpośrednie koszty usługi gastronomicznej » ('Le food cost désigne en effet des frais directs d'un service gastronomique $)^{21}$.

Food justice, trouvé une fois dans la presse imprimée, n’apparaît que sur les sites anglophones. La citation contient l'explication du terme :

Obowiązkowym przedmiotem w tym roku jest food justice - uczą się o produkcji i dystrybucji jedzenia w świecie i tego, dlaczego, gdy my wyrzucamy połowę jedzenia, inne dzieci są głodne » ('La matière obligatoire cette année

19 « Organizacja Foodharing [sic] Kraków zaprasza dziś o godz. 19 [...] na otwarcie pierwszej krakowskiej Jadłodzielni. Zapraszamy każdego, któremu bliska jest idea dzielenia się jedzeniem, aby przyniósł ze sobą artykuł spożywczy, który zostawi w jadłodzielni » ('Lorganisation Foodsharing Cracovie invite ce soir à 19h à l'inauguration de la première Jadłodzielnia de Cracovie. Nous invitons chacun à qui l'idée du partage de la nourriture est chère à apporter un produit alimentaire qu'il laissera dans la jadłodzielnia') dans Dziennik Polski, le 23 septembre 2016 et plusieurs fois dans un article de Polityka du 12 octobre 2016 : « Foodsharing Warszawa : Jadłodzielnia ma działać na zasadzie odruchu: daję - biorę " ('Le foodsharing doit fonctionner selon le principe du réflexe : je donne - je prends'). «Do foodsharingu (z ang. food - jedzenie, share - dzielić) wystarczy niewiele [...]» ('Pour faire fonctionner le foodsharing, de l'ang. food - nourriture, share - partager, il ne faut pas grand-chose').

20 « [...] od kelnerskich machlojek, przez food cost i opisanie kuchennych stanowisk [...] " ('en partant des magouilles de serveurs, par le food cost et la description de tâches à la cuisine') dans Gazeta Wyborcza du 9 juillet 2016.

21 Un autre, http://gastrowiedza.pl/baza-wiedzy/slownik-hasel/f/food-cost-z-angkoszt-zywnosci-inaczej-koszt-surowca_apporte une note avec un titre et sa traduction en polonais « Food cost (z ang. koszt żywności, inaczej koszt surowca)». 
c'est food justice - on fait prendre connaissance de la production et de la distribution de la nourriture dans le monde et pourquoi, quand nous jetons la moitié de notre nourriture, d'autres enfants souffrent de la faim').

L'intérêt porté à la cuisine se manifeste, comme nous le savons, par la présence des sites, des blogs, des présentations vidéo. Dans ce cadre, le mot food writer 'chroniqueur culinaire' et son équivalent polonais krytyk kulinarny se trouvent fréquemment. Le contexte ${ }^{22}$ le donne au féminin, régulièrement formé à partir de l'original anglais: food writerka. Les deux formes anglaises semblent être absentes des sites en polonais.

Good food ne figure qu'une fois ${ }^{23}$ dans le corpus. La citation laisse comprendre qu'il s'agit d'un mot nouveau : «Tę sprzeczność szybkiego slow food na świecie rozwiązano za pomocą nowego określenia: good food, czyli po prostu dobre jedzenie. » ('Cette contradiction du slow food rapide a été éliminée à l'aide d'un nouveau terme: good food, simplement une bonne nourriture'). Good food est utilisé aussi comme nom de restaurants ou marque de produits alimentaires, (celle p. ex. de gaufres de riz).

Un terme encore, peasant food, apparaît une fois ${ }^{24}$ dans le corpus, visiblement par référence à la cuisine anglaise, mais utilisé consciemment, avec un commentaire en polonais : «Droga pani, to nie jest british [sic] cuisine. To peasant food, jedzenie dla wieśniaków» ('Chère madame, ce n'est pas de la British cuisine, c'est du peasant food, nourriture de ploucs'). Le terme n'apparaît pas dans les contextes polonais sur le web, en anglais sa signification est définie comme la cuisine des pauvres, surtout à la campagne.

Raw food figure une seule fois ${ }^{25}$ dans le corpus :

Dieta raw food opiera się głównie na produktach roślinnych. Obejmuje świeże owoce i warzywa, kiełki, orzechy, nasiona, ziarna - w jej skład mogą wejść również owoce morza, mięso, jajka, mleko itp. (w zależności od poglądów) ('Le régime raw food est fondé surtout sur les produits végétaux. Il contient légumes et fruits frais, pousses, noix, graines - il admet aussi les fruits de mer, la viande, les œufs, le lait, etc., en fonction des convictions individuelles').

22 «Rok temu przestrzeń wynajęła para - szef kuchni i food writerka " ('Il y a un an l'espace a été loué par un couple - un chef de cuisine et une food writer'), Gazeta Wyborcza, le 20 août 2016.

23 Polityka, le 13 mai 2016.

24 Gazeta Wyborcza, le 2 juillet, 2016.

25 Gazeta Wyborcza - Duży Format, le 21 juillet 2016. 
Le terme est présent sur internet à partir de 2005.

Soul food est employé plusieurs fois dans un article de Polityka ${ }^{26}$, avec la traduction strawa duchowa 'nourriture spirituelle', ou jedzenie pocieszenia 'nourriture de consolation'. Wikipédia version polonaise définit le soul food comme une cuisine de la population noire du sud des États-Unis, datant de l'époque de l'esclavage et se caractérisant par des ingrédients simples et bon marché. Soul food est aussi le titre d'un feuilleton télévisé de 2000, connu en Pologne sous ce titre. Dans l'article cité de Polityka, on lit :

Pewnego dnia stwierdził, że skoro on jest „królem soulu”, to jego babcia będzie królową soul foodu. (...). dzięki temu o soul food usłyszeli wszyscy" ('Un jour il a décidé que puisqu'il était « le roi de la soul», sa grand-mère serait la reine de la soul food (...), grâce à cela, tous ont entendu parler de la soul food')

ou encore :

«Soul food też jest takim jedzeniem pocieszenia » ('La soul food est aussi une telle nourriture de consolation'), et une remarque, toujours dans le même article : « (...) W polskich miastach powstaje coraz więcej lokali i food trucków ze słowami soul food w nazwie " ('Dans les villes polonaises, on ouvre de plus en plus de restaurants et de food trucks avec des noms comportant soul food').

Street food apparaît également dans des formes où le deuxième élément est décliné : street foodu, street foodem ou comme base de l'adjectif streetfoodowy, décliné lui aussi. Dans une des cinq citations, on trouve la définition suivante du terme : «Dzisiaj nazwalibyśmy je street foodem, prostym jedzeniem które można było kupić na każdym rogu » ('Aujourd'hui on l'appellerait street food, une nourriture simple à acheter à chaque coin de rue') 27 .

Nous voudrions ajouter à l'inventaire d'emprunts avec food quelques noms de restaurants ou d'évènements gastronomiques dont l'intitulé comporte un anglicisme, certainement pour le prétendu prestige. Ont été relevés : strefa Slow Food, Festiwal Street food, plusieurs noms avec food truck: Food Truck Spot Bonarka, Bezogródek Food Truck Park, Przystań Foodtruck, Manufaktura Krakowska Foodtruck, restaurant Good Food. Et enfin un exemple relevé sur le site de l'Observatoire de Varsovie : „Food Patrol” powstał z pasji do jedzenia i jest pierwszym food truckiem serwującym hamburgery w Poznaniu ('Food Patrol

26 Du 20 avril 2016, article "Strawa duchowa ».

27 Gazeta Wyborcza, le 24 septembre 2016. 
est né d'une passion culinaire et il est le premier food truck qui sert des hamburgers à Poznań').

Pour récapituler ces quelques remarques sur les néologismes avec food en polonais, nous voudrions observer que, quoiqu' il soit difficile de dater leur apparition exacte dans cette langue, nous pouvons avancer qu' ils se trouvent dans les textes polonais, pour la plupart, certainement à partir du début des années 2000. Les composés avec food s'adaptent à la flexion polonaise en prenant pour l'élément food les formes des cas, de la déclinaison masculine ou neutre. Ils forment le pluriel selon la morphologie polonaise et servent à la formation des adjectifs ou plus rarement des substantifs (fastfoodztwo). La graphie - soudée ou séparée - n'est pas encore stabilisée en polonais. Toutefois, les adjectifs utilisent le composé en forme liée. La prononciation semble être proche de la langue d'origine, au moins pour les mots les plus répandus. Quelques emprunts ont déjà leurs équivalents, fonctionnant en polonais depuis longtemps : bar szybkiej obstugi-fast food 'restaurant', krytyk kulinarny - food writer, ou bien créés pratiquement au moment de l'introduction du néologisme anglais : jadłobus, jadłowóz, gastrobus pour foodtruck; jadłodzielnia pour foodsharing, śmieciowe jedzenie pour junk food. Il s'agit de calques ou de compositions plus ou moins réussies.

Les formes présentées ci-dessus ${ }^{28}$ apparaissent dans les textes avec une fréquence variée, certaines sont répertoriées dans l'Observatoire linguistique qui pourra servir plus tard à la documentation lexicographique, un seul (fast food) est noté par le dictionnaire le plus récent (WSJP online). Même s'il est difficile à présent d'évaluer leur chance d'entrer réellement dans le lexique polonais, il importe de les signaler dès maintenant.

\section{Références bibliographiques}

Bochnakowa Anna, Hildenbrand Zuzana, 2016, «Des néologismes culinaires récents en polonais et en tchèque ", in Emprunts néologiques et équivalents autochtones en français, en polonais et en tchèque, Zuzana Hildenbrand, Alicja Kacprzak, Jean-François Sablayrolles (éds.), Limoges, Lambert Lucas, coll. « La Lexicothèque », p. 227-266.

Hildenbrand Zuzana, Jacquet-Pfau Christine, 2016, « Les pratiques alimentaires, un domaine très ouvert aux emprunts », in Emprunts néologiques et équivalents autochtones en français, en polonais et en tchèque, Zuzana

28 Leur inventaire varie en quelques points de celui que Christine Jacquet-Pfau et Zuzana Hildenbrand avaient dressé dans leur article. 
Anna BOCHNAKOWA

Hildenbrand, Alicja Kacprzak, Jean-François Sablayrolles (éds.), Limoges, Lambert Lucas, coll. « La Lexicothèque », p. 175-225.

NKJP - Narodowy Korpus Języka Polskiego, http://nkjp.pl/.

Obserwatorium Językowe UW, http://nowewyrazy.uw.edu.pl/ (août- décembre 2016).

WSJP - Wielki słownik języka polskiego PAN, 2007, Piotr. Żmigrodzki (éd.), Kraków, Instytut Języka Polskiego PAN, http://www.wsjp.pl (août - décembre 2016). 